\title{
On solvability of a nonlocal problem for the Laplace equation with the fractional-order boundary operator
}

\author{
Moldir A Muratbekova*, Kanat M Shinaliyev and Batirkhan K Turmetov
}

"Correspondence:

moldir_1983@mail.ru Department of Mathematics,

Akhmet Yasawi International Kazakh-Turkish University, B. Sattarkhanov Street 29, Turkistan, 161200, Kazakhstan

\begin{abstract}
In the present work, we study properties of some integro-differential operators of the Hadamard-Marchaud type in the class of harmonic functions. As an application of these properties, we consider the question of the solvability of a nonlocal boundary value problem for the Laplace equation in the unit ball.

MSC: $35 \mathrm{~J} 05 ; 35 \mathrm{~J} 25 ; 26 \mathrm{~A} 33$
\end{abstract}

Keywords: Hadamard-Marchaud operator; fractional derivative; nonlocal problem

\section{Introduction}

Let $\Omega=\left\{x \in R^{n}:|x|<1\right\}$ be the unit ball, $n \geq 2$. A paradigm in the theory of elliptic partial differential equations and harmonic functions is the Laplace equation

$$
\Delta u(x)=0, \quad x \in \Omega
$$

If we prescribe the values of the solution at the boundary $\partial \Omega$ of $\Omega$, then we can solve equation (1) uniquely. Of course, one can consider many other boundary conditions such as Neumann's boundary conditions.

In some applied problems of hydrodynamics [1], it is necessary to prescribe the value of a fractional derivative of the solution on the boundary. Fractional differential equations and boundary value problems involving fractional derivatives appear in many applied problems ranging from the spring-pot model [2] to geology [3] or from nonlinear circuits [4] to alternative models to differential equations [5].

Hence, in this paper we study the Laplace equation concentrating on some conditions on the boundary involving derivatives of fractional order.

Note that numerous works of authors [6-13] were dedicated to the research questions of the solvability of boundary value problems for partial differential equations with boundary operators of high (whole and fractional) order. In the paper of A.N. Tikhonov [14] boundary value problems with boundary conditions containing derivatives of higher order have been investigated for the heat equation. Research questions as regards the solvability of similar problems for higher-order equations with boundary operators of whole and fractional order were carried out in $[15,16]$. Later in [17], these results were generalized for partial differential equations of fractional order. In [18-20] questions about the solvability 
of boundary value problems with boundary operators of high order were studied for the Laplace equation. In the studies of these authors the exact conditions for the solvability have been established and the integral representations of solutions of the studied problems have been found. The cycle of studies by the authors [21-26] is devoted to the study of the existence and smoothness of solutions of boundary value problems for the second-order elliptic equations with boundary operators of fractional order. In the paper mentioned above local boundary value problems with boundary operators of fractional order in the Riemann-Liouville or Caputo sense are studied. In this paper we study nonlocal problems with boundary operators of fractional-order derivatives of Hadamard type. Definitions of Hadamard operators, a statement of the main problems, and the history of the questions on this topic are in Section 3.

The organization of this paper is as follows. In Section 2, we present the operators of integration and differentiation in the Hadamard sense and some modifications. In the third section we provide a formulation of the basic problem of this paper and some historical information as regards nonlocal boundary value problems. In the fourth section we study the properties of integral and differential Hadamard-Marchaud operators in the class of harmonic functions in the ball. In Section 5 we provide some auxiliary propositions. Finally, Section 6 is devoted to the study of the fundamental problem, where we formulate and prove the main statement of the paper.

\section{Definition of Hadamard operators of integration and differentiation and some modifications}

In this section, we give a statement on the operators of fractional differentiation in the sense of Hadamard, Hadamard-Marchaud, and their modifications.

For any positive $\alpha$, fractional integrals and derivatives of the order $\alpha$ in the sense of Hadamard are defined by the following formulas [27]:

$$
\begin{aligned}
& J^{\alpha} \varphi(t)=\frac{1}{\Gamma(\alpha)} \int_{0}^{t}\left(\ln \frac{t}{s}\right)^{-(\alpha+1)} \frac{\varphi(s)}{s} d s, \quad t>0, \\
& D^{\alpha} \varphi(t)=\delta^{m+1} J^{m+1-\alpha} \varphi(t),
\end{aligned}
$$

where $\delta=t \frac{d}{d t}$ is the Dirac operator, $m=[\alpha]$ is the integral part of $\alpha$.

If $0<\alpha<1$, then, in the class of sufficiently 'good' functions, operator (3) can be reduced to the following form [27]:

$$
D^{\alpha} \varphi(t)=\delta J^{1-\alpha} \varphi(t)=\frac{\alpha}{\Gamma(1-\alpha)} \int_{0}^{1} \frac{\varphi(t)-\varphi(s t)}{s|\ln s|^{\alpha+1}} d s
$$

This operator is said to be the differentiation operator of order $\alpha$ in the sense of Hadamard-Marchaud.

In [28], the following modification of the Hadamard-Marchaud operator was considered:

$$
D_{\mu}^{\alpha} \varphi(t)=\frac{\alpha}{\Gamma(1-\alpha)} \int_{0}^{1} \frac{\varphi(t)-\varphi(s t)}{s^{1-\mu}|\ln s|^{\alpha+1}} d s+\mu^{\alpha} \varphi(t), \quad \mu \geq 0 .
$$


In [18], in the class of harmonic functions in a ball, the properties and applications of the operators in the form of

$$
\delta_{\mu}=r \frac{\partial}{\partial r}+\mu, \quad \delta_{\mu}^{m}=\left(r \frac{\partial}{\partial r}+\mu\right)^{m}
$$

are considered. Here $\mu>0, r=|x|, x=\left(x_{1}, \ldots, x_{n}\right)$, and $r \frac{\partial}{\partial r}$ is a differential operator in the form of $r \frac{\partial}{\partial r}=\sum_{i=1}^{n} x_{i} \frac{\partial}{\partial x_{i}}$.

Let $u(x)$ be a harmonic function in the domain $\Omega$, and let $0 \leq \alpha, 0<\mu$ be arbitrary real numbers. Let us consider a modification of the Bavrin operator (6).

Introduce the operators

$$
\begin{aligned}
& J_{\mu}^{\alpha}[u](x)= \begin{cases}u(x), & \alpha=0, \\
\frac{1}{\Gamma(\alpha)} \int_{0}^{1}|\ln s|^{\alpha-1} s^{\mu-1} u(s x) d s, & \alpha>0,\end{cases} \\
& D_{\mu}^{\alpha}[u](x)= \begin{cases}u(x), & \alpha=0, \\
\frac{\alpha}{\Gamma(1-\alpha)} \int_{0}^{1} \frac{u(x)-u(s x)}{s^{1-\mu}|\ln s|^{\alpha+1}} d s+\mu^{\alpha} u(x), & 0<\alpha<1, \\
\left(r \frac{\partial}{\partial r}+\mu\right)^{m} D_{\mu}^{\alpha-m}[u](x), & m \leq \alpha<m+1, m=1,2, \ldots .\end{cases}
\end{aligned}
$$

If $\alpha=m, \mu>0$, then we obtain the Bavrin operator $D_{\mu}^{m}[u](x)=\left(r \frac{\partial}{\partial r}+\mu\right)^{m}$.

\section{Statement of the problem}

Let $t_{k}: \partial \Omega \rightarrow \Gamma_{k} \subset \bar{\Omega}_{1} \subset \Omega, k=1,2, \ldots, \Gamma_{k} \neq \emptyset$, be continuous mappings, and let $a_{k}(x)$ be continuous functions satisfying the condition

$$
\sum_{k=1}^{\infty}\left|a_{k}(x)\right|<\infty, \quad x \in \partial \Omega
$$

We assume that the series (7) converges uniformly on $\partial \Omega$.

Further, let $\mu>0,0 \leq \beta \leq \alpha$, and $\alpha+\beta \neq 0$, i.e. $\alpha$ and $\beta$ are not equal to zero simultaneously.

Consider the following boundary value problem:

$$
\begin{aligned}
& \Delta u(x)=0, \quad x \in \Omega, \\
& D_{\mu}^{\alpha}[u](x)-\sum_{k=1}^{\infty} a_{k}(x) D_{\mu}^{\beta}[u]\left(t_{k}(x)\right)=f(x), \quad x \in \partial \Omega,
\end{aligned}
$$

where $f(x) \in C(\partial \Omega)$.

A harmonic function $u(x)$ from the class $C^{2}(\Omega) \cap C(\bar{\Omega})$, such that $D_{\mu}^{\alpha} u(x) \in C(\bar{\Omega})$ and condition (9) is realized in the classical sense, will be called a solution of problem (8)-(9).

The above-mentioned problem is a simple generalization of Bitsadze-Samarskii's nonlocal problem [29]. For convenience of the reader, we formulate the Bitsadze-Samarskii problem.

Let $D$ be a finite simply-connected domain of the plane of complex variables $z=x+i y$ with the smooth boundary $S=\partial D$, and let $S_{1}$ be a closed simple smooth curve lying in $D$.

We denote by $\tau=v(t), t \in S, \tau \in S_{1}$ a diffeomorphism between $S$ and $S_{1}$. 
Formulation of the problem: We are to find a harmonic function $u(z)=u(x, y)$ in $D$, which is continuous in $\bar{D}=D \cup S$ and satisfies the boundary condition

$$
u(t)-u(v(t))=f(t), \quad t \in S,
$$

where $f(t)$ is a given function.

Similar problems with operators of integer order were considered in [30-32], and for operators of fractional order with fractional-order derivatives in the sense of RiemannLiouville and Caputo in [33-41]. It should also be noted that some questions of solvability of nonlocal problems for fractional-order equations in the one-dimensional case were studied in [42-44].

\section{Properties of operators $J_{\mu}^{\alpha}$ and $D_{\mu}^{\alpha}$}

In this section, we study some properties of the operators $J_{\mu}^{\alpha}$ and $D_{\mu}^{\alpha}$ in the class of harmonic functions. Further, for convenience, we shall take everywhere $\gamma=\alpha-m$.

Lemma 1 Let $\alpha>0, \mu>0$, and $H_{k}(x)$ be a homogeneous harmonic polynomial of the power $k \in N_{0}=\{0,1, \ldots\}$. Then the following equalities are correct:

$$
\begin{array}{ll}
J_{\mu}^{\alpha}\left[H_{k}\right](x)=(k+\mu)^{-\alpha} H_{k}(x), & \mu>0, k \in N_{0}, \\
D_{\mu}^{\alpha}\left[H_{k}\right](x)=(k+\mu)^{\alpha} H_{k}(x), & k \in N_{0}, \mu>0 .
\end{array}
$$

Proof Let $\mu>0$. Then, using homogeneity of the polynomial $H_{k}(x)$, we obtain

$$
J_{\mu}^{\alpha}\left[H_{k}\right](x)=\frac{1}{\Gamma(\alpha)} \int_{0}^{1}|\ln s|^{\alpha-1} s^{\mu-1} H_{k}(s x) d s=\frac{H_{k}(x)}{\Gamma(\alpha)} \int_{0}^{1}|\ln s|^{\alpha-1} s^{k+\mu-1} d s .
$$

The value of the last integral can easily be calculated with the help of the change of variables $z=-\ln s$. In fact,

$$
\begin{aligned}
\frac{1}{\Gamma(\alpha)} \int_{0}^{1}|\ln s|^{\alpha-1} s^{k+\mu-1} d s & =\frac{1}{\Gamma(\alpha)} \int_{0}^{\infty} z^{\alpha-1} e^{-(k+\mu) z} d z \\
& =\frac{(k+\mu)^{-\alpha}}{\Gamma(\alpha)} \int_{0}^{\infty} t^{\alpha-1} e^{-t} d t=(k+\mu)^{-\alpha}
\end{aligned}
$$

The equality (10) is proved.

Further, note that the relation

$$
\delta_{\mu}^{m} H_{k}(x) \equiv\left(r \frac{\partial}{\partial r}+\mu\right)^{m} H_{k}(x)=(k+\mu)^{m} H_{k}(x), \quad k \in N_{0}, \mu \geq 0,
$$

holds for the operator $\delta_{\mu}^{m}$.

Now, let us study actions of the operator $D_{\mu}^{\gamma}$ to the functions $H_{k}(x)$. Using the definition of $D_{\mu}^{\gamma}$ and the homogeneity of $H_{k}(x)$, we have

$$
\begin{aligned}
D_{\mu}^{\gamma}\left[H_{k}\right](x) & =\frac{\gamma}{\Gamma(1-\gamma)} \int_{0}^{1} \frac{H_{k}(x)-H_{k}(s x)}{s^{1-\mu}|\ln s|^{\gamma+1}} d s+\mu^{\gamma} H_{k}(x) \\
& =\frac{\gamma H_{k}(x)}{\Gamma(1-\gamma)} \int_{0}^{1}\left(s^{\mu-1}-s^{k+\mu-1}\right)|\ln s|^{-(\gamma+1)} d s+\mu^{\gamma} H_{k}(x) .
\end{aligned}
$$


Denoting $\mathrm{I}=\int_{0}^{1}\left(s^{\mu}-s^{k+\mu}\right)\left(\ln \frac{1}{s}\right)^{-(\gamma+1)} \frac{d s}{s}$ and integrating by parts, we get

$$
\mathrm{I}=-\frac{1}{\gamma} \int_{0}^{1}\left[\mu s^{\mu-1}-(k+\mu) s^{k+\mu-1}\right]\left(\ln \frac{1}{s}\right)^{-\gamma} d s
$$

After the change of variables $z=-\ln s$, as in the proof of equality (10), we easily obtain

$$
\mathrm{I}=-\frac{\Gamma(1-\gamma)}{\gamma} \mu^{\gamma}+\frac{\Gamma(1-\gamma)}{\gamma}(k+\mu)^{\gamma}
$$

which implies $D_{\mu}^{\gamma}\left[H_{k}\right](x)=(k+\mu)^{\gamma} H_{k}(x)$.

Further, taking into account fulfilling of equality (12), we obtain in the general case for $m \leq \alpha<m+1$ :

$$
D_{\mu}^{\alpha}\left[H_{k}\right](x)=\delta_{\mu}^{m} D_{\mu}^{\gamma}\left[H_{k}\right](x)=(k+\mu)^{\alpha} H_{k}(x) .
$$

The lemma is proved.

Lemma 2 Let $\alpha>0, \mu>0$, and $u(x)$ be a harmonic function in the ball $\Omega$. Then the functions $D_{\mu}^{\alpha}[u](x)$ and $J_{\mu}^{\alpha}[u](x)$ are also harmonic in $\Omega$.

Proof Let $u(x)$ be a harmonic function in the ball $\Omega$. Then it is known [45] that the function $u(x)$ is represented in the form of the series

$$
u(x)=\sum_{k=0}^{\infty} \sum_{i=1}^{H_{k}} u_{k}^{(i)} H_{k}^{(i)}(x)
$$

where $\left\{H_{k}^{(i)}(x): i=1, \ldots, h_{k}\right\}$ is a complete system of homogeneous harmonic polynomials of power $k$, and $u_{k}^{(i)}$ are coefficients of the expansion (13). Applying formally the operator $D_{\mu}^{\alpha}$ to the series (13) and taking into account equality (11), we obtain

$$
D_{\mu}^{\alpha}[u](x)=\sum_{k=0}^{\infty} \sum_{i=1}^{H_{k}}(k+\mu)^{\alpha} u_{k}^{(i)} H_{k}^{(i)}(x) .
$$

Now let us check convergence of the series (13) and (14). The following asymptotical estimate is valid for $h_{k}$ :

$$
h_{k}=\left(1+\frac{2 k}{(n-2)}\right) C_{k+n-3}^{n-3} \approx \frac{2 k^{n-2}}{(n-2) !}, \quad k \rightarrow \infty(n \geq 3) .
$$

Moreover, the series (13) converges absolutely and uniformly by $x$ at $|x| \leq \rho<1$, hence, for any $|x| \leq \rho$ and any $i, k \in N_{0}$, the equalities $\left|u_{k}^{(i)} H_{k}^{(i)}(x)\right| \leq c_{\rho}$ hold. Since $\lim _{k \rightarrow \infty} \sqrt[k]{(k+\mu)^{\alpha}}=1$, we have for $|x| \leq r \rho$ and $r<1$

$$
\sum_{k=0}^{\infty} \sum_{i=1}^{h_{k}}(k+\mu)^{\alpha}\left|u_{k}^{(i)} H_{k}^{(i)}(x)\right| \leq c_{\rho} \sum_{k=0}^{\infty}\left[(k+\mu)^{\alpha} h_{k}\right] r^{k}<\infty
$$

Therefore, the series (14) converges absolutely and uniformly by $x$ at $|x| \leq r \rho$, where $r<1, \rho<1$, and its sum is a harmonic function. By virtue of the arbitrariness of $r<1$ and $\rho<1$, the function $D_{\mu}^{\alpha}[u](x)$ is defined in the whole ball $\Omega$. 
Let us study the function $J_{\mu}^{\alpha}$. Applying formally the operator $J_{\mu}^{\alpha}$ to the series (13), taking into account equality (10), we obtain

$$
J_{\mu}^{\alpha}[u](x)=\sum_{k=1}^{\infty} \sum_{i=1}^{h_{k}}(k+\mu)^{-\alpha} u_{k}^{(i)} H_{k}^{(i)}(x) .
$$

Convergence of this series can be checked as in the case of series (14), and that is why $J_{\mu}^{\alpha}[u](x)$ is a harmonic function in the ball $\Omega$. The lemma is proved.

Now we show that the function $u(x)$ can be represented in terms of the function $D_{\mu}^{\alpha}[u](x)$.

Lemma 3 Let $\alpha>0, \mu>0$, and $u(x)$ be a harmonic function in the domain $\Omega$. Then for any $x \in \Omega$ the equality

$$
u(x)=\frac{1}{\Gamma(\alpha)} \int_{0}^{1}|\ln s|^{\alpha-1} s^{\mu-1} D_{\mu}^{\alpha}[u](s x) d s
$$

is valid.

Proof Let $\mu>0$. Represent a harmonic function $u(x)$ in the form of the series (13) and transform it to the form of

$$
u(x)=\sum_{k=0}^{\infty} \sum_{i=1}^{h_{k}}(k+\mu)^{\alpha} \frac{u_{k}^{(i)}}{(k+\mu)^{\alpha}} H_{k}^{(i)}(x) .
$$

Further, taking into account equalities (10)-(11), and the absolute and uniform convergence of the series (15) by $x$ at $|x| \leq \rho<1$, it can be reduced to the form of

$$
\begin{aligned}
u(x) & =\sum_{k=0}^{\infty} \sum_{i=1}^{h_{k}}(k+\mu)^{\alpha} \frac{u_{k}}{\Gamma(\alpha)} \int_{0}^{1}|\ln s|^{\alpha-1} s^{\mu-1} H_{k}^{(i)}(s x) d s \\
& =\frac{1}{\Gamma(\alpha)} \int_{0}^{1}|\ln s|^{\alpha-1} s^{\mu-1} D_{\mu}^{\alpha}\left[\sum_{k=0}^{\infty} \sum_{i=1}^{h_{k}} u_{k}^{(i)} H_{k}^{(i)}(s x)\right] d s \\
& =\frac{1}{\Gamma(\alpha)} \int_{0}^{1}|\ln s|^{\alpha-1} s^{\mu-1} D_{\mu}^{\alpha}[u](s x) d s .
\end{aligned}
$$

The lemma is proved.

One can similarly prove the following lemma.

Lemma 4 Let $0<\beta<\alpha, \mu>0$, and $u(x)$ be a harmonic function in the domain $\Omega$. Then for any $x \in \Omega$ the equality

$$
D_{\mu}^{\beta}[u](x)=\frac{1}{\Gamma(\alpha-\beta)} \int_{0}^{1}|\ln s|^{\alpha-\beta-1} s^{\mu-1} D_{\mu}^{\alpha}[u](s x) d s
$$

is valid. 
Lemma 5 Let $\alpha>0, \mu>0$, and $u(x)$ be a harmonic function in the domain $\Omega$. Then the following equalities hold:

$$
J_{\mu}^{\alpha}\left[D_{\mu}^{\alpha}[u]\right](x)=D_{\mu}^{\alpha}\left[J_{\mu}^{\alpha}[u]\right](x)=u(x) .
$$

Proof Let $\mu>0$. Applying the operator $J_{\mu}^{\alpha}$ to the function $D_{\mu}^{\alpha}[u](x)$, we obtain

$$
J_{\mu}^{\alpha}\left[D_{\mu}^{\alpha}[u]\right](x)=\frac{1}{\Gamma(\alpha)} \int_{0}^{1}|\ln s|^{\alpha-1} s^{\mu-1} D_{\mu}^{\alpha}[u](s x) d s .
$$

By virtue of Lemma 3, the value of the last integral is equal to $u(x)$, i.e. $J_{\mu}^{\alpha}\left[D_{\mu}^{\alpha}[u]\right](x)=u(x)$. To prove the second equality, apply the operator $D_{\mu}^{\gamma}$ to the function $J_{\mu}^{\alpha}[u](x)$. We get

$$
\begin{aligned}
D_{\mu}^{\gamma}\left[J_{\mu}^{\alpha}[u]\right](x) & =\frac{\gamma}{\Gamma(1-\gamma)} \int_{0}^{1} \frac{J_{\mu}^{\alpha}[u](x)-J_{\mu}^{\alpha}[u](s x)}{s^{1-\mu} \ln |s|^{\gamma+1}} d s+\mu^{\gamma} J_{\mu}^{\alpha}[u](x) \\
& =\frac{\gamma}{\Gamma(1-\gamma)} \int_{0}^{1} \frac{1}{s^{1-\mu} \ln |s|^{\gamma+1}} \frac{1}{\Gamma(\alpha)} \int_{0}^{1} \frac{u(\tau x)-u(\tau s x)}{\tau^{1-\mu} \ln |\tau|^{1-\alpha}} d \tau d s+\mu^{\gamma} J_{\mu}^{\alpha}[u](x) \\
& =\frac{1}{\Gamma(\alpha)} \int_{0}^{1} \frac{1}{|\ln \tau|^{1-\alpha} \tau^{1-\mu}} \frac{\gamma}{\Gamma(1-\gamma)} \int_{0}^{1} \frac{u(\tau x)-u(\tau s x)}{|\ln s|^{(\gamma+1)} s^{1-\mu}} d s d \tau+\mu^{\gamma} J_{\mu}^{\alpha}[u](x) \\
& =\frac{1}{\Gamma(\alpha)} \int_{0}^{1} \frac{D_{\mu}^{\gamma}[u](\tau x)-\mu^{\gamma} u(\tau x)}{|\ln \tau|^{1-\alpha} \tau^{1-\mu}} d \tau+\mu^{\gamma} J_{\mu}^{\alpha}[u](x) \\
& =\frac{1}{\Gamma(\alpha)} \int_{0}^{1} \frac{D_{\mu}^{\gamma}[u](\tau x) d \tau}{|\ln \tau|^{1-\alpha} \tau^{1-\mu}}-\mu^{\gamma} \frac{1}{\Gamma(\alpha)} \int_{0}^{1} \frac{u(\tau x) d \tau}{|\ln \tau|^{1-\alpha} \tau^{1-\mu}}+\mu^{\gamma} J_{\mu}^{\alpha}[u](x) \\
& =\frac{1}{\Gamma(\alpha)} \int_{0}^{1} \frac{D_{\mu}^{\gamma}[u](\tau x)}{|\ln \tau|^{1-\alpha} \tau^{1-\mu}} d \tau-\mu^{\gamma} J_{\mu}^{\alpha}[u](x)+\mu^{\gamma} J_{\mu}^{\alpha}[u](x) \\
& =\frac{1}{\Gamma(\alpha)} \int_{0}^{1} \frac{D_{\mu}^{\gamma}[u](\tau x)}{|\ln \tau|^{1-\alpha} \tau^{1-\mu}} d \tau .
\end{aligned}
$$

Then, in the general case,

$$
\begin{aligned}
D_{\mu}^{\alpha}\left[J_{\mu}^{\alpha}[u]\right](x) & =\delta_{\mu}^{m} D_{\mu}^{\gamma}\left[J_{\mu}^{\alpha}[u]\right](x)=\frac{1}{\Gamma(\alpha)} \int_{0}^{1} \frac{\delta_{\mu}^{m} D_{\mu}^{\gamma}[u](\tau x)}{|\ln \tau|^{1-\alpha} \tau^{1-\mu}} d \tau \\
& =\frac{1}{\Gamma(\alpha)} \int_{0}^{1}|\ln \tau|^{\alpha-1} \tau^{\mu-1} D_{\mu}^{\alpha}[u](\tau x) d \tau=u(x) .
\end{aligned}
$$

The lemma is proved.

\section{Some auxiliary propositions}

Let $t_{k}$ and $a_{k}(x)$ satisfy the conditions from Section 2 .

Consider the following problem in the domain $\Omega$ :

$$
\begin{aligned}
& \Delta u(x)=0, \quad x \in \Omega, \\
& v(x)-\sum_{k=1}^{\infty} a_{k}(x) J_{\mu}^{\alpha-\beta}[u]\left(t_{k}(x)\right)=f(x), \quad x \in \partial \Omega,
\end{aligned}
$$

where $\mu>0,0 \leq \beta \leq \alpha$, and $\alpha+\beta \neq 0$, i.e. $\alpha$ and $\beta$ are not equal to zero simultaneously, $f(x) \in C(\partial \Omega)$. 
A harmonic function $v(x)$ from the class $C^{2}(\Omega) \cap C(\bar{\Omega})$, satisfying condition (18) in the classical case, will be called a solution of problem (17)-(18).

It should be noted that problem (17)-(18) was investigated for the case of $\alpha=\beta$ in [30]. Let us investigate uniqueness for the solution of problem (17)-(18). The following statement holds.

Lemma 6 Let $\Gamma_{k} \subset \bar{\Omega}_{1} \subset \Omega, k=1,2, \ldots, a_{k}(x), k=0,1,2, \ldots$, be continuous functions satisfying the condition

$$
\sum_{k=1}^{\infty}\left|a_{k}(x)\right| \leq \mu^{\alpha-\beta}, \quad x \in \partial \Omega
$$

and let a solution of problem (17)-(18) exist.

Then:

(1) If

$$
\sum_{k=1}^{\infty} a_{k}(x) \neq \mu^{\alpha-\beta}, \quad x \in \partial \Omega
$$

then the solution of problem (17)-(18) is unique.

(2) If

$$
\sum_{k=1}^{\infty} a_{k}(x) \equiv \mu^{\alpha-\beta}, \quad x \in \partial \Omega
$$

then the solution of problem (17)-(18) is unique up to a constant summand.

Proof Let $v(x)$ be the solution of problem (17)-(18) at $f(x)=0$.

Denote $M=\left|v\left(x_{0}\right)\right|=\max _{\partial \Omega}|v(x)|, x_{0} \in \partial \Omega$.

Then if $v(x) \neq$ const, then, by virtue of the maximum principle for harmonic functions [46], the inequality $|v(x)|<M$ holds for any $x \in \Omega$.

The boundary condition (18) at $f(x)=0$ implies

$$
\begin{aligned}
M & =\left|\sum_{k=1}^{\infty} a_{k}\left(x_{0}\right) J_{\mu}^{\alpha-\beta}[v]\left[t_{k}\left(x_{0}\right)\right]\right| \leq \sum_{k=1}^{\infty}\left|a_{k}\left(x_{0}\right)\right|\left|J_{\mu}^{\alpha-\beta}[v]\left(t_{k}\left(x_{0}\right)\right)\right| \\
& \leq \sum_{k=1}^{\infty}\left|a_{k}\left(x_{0}\right)\right| \frac{1}{\Gamma(\alpha-\beta)} \int_{0}^{1} s^{\mu-1}|\ln s|^{(\alpha-\beta-1)}\left|v\left(s t_{k}\left(x_{0}\right)\right)\right| d s .
\end{aligned}
$$

Further, since $t_{k}: \partial \Omega \rightarrow \Gamma_{k} \subset \bar{\Omega}_{1} \subset \Omega, k=1,2, \ldots, \Gamma_{k} \neq \emptyset$, then $t_{k}\left(x_{0}\right) \in \Omega$, and for any $s \in[0,1], s t_{k}\left(x_{0}\right) \in \Omega$. Therefore $\left|v\left(s t_{k}\left(x_{0}\right)\right)\right|<M$.

Hence,

$$
M<M \sum_{k=1}^{\infty}\left|a_{k}\left(x_{0}\right)\right| \frac{1}{\Gamma(\alpha-\beta)} \int_{0}^{1} s^{\mu-1}|\ln s|^{\alpha-\beta-1} d s=M \mu^{\beta-\alpha} \sum_{k=1}^{\infty}\left|a_{k}\left(x_{0}\right)\right| .
$$

If now condition (19) is realized, then $\mu^{\beta-\alpha} \sum_{k=1}^{\infty}\left|a_{k}\left(x_{0}\right)\right| \leq 1$, and we obtain from this the contradiction $M<M$. 
Hence, if condition (19) holds, it is necessary that $v(x)=C \equiv$ const. Since $J_{\mu}^{\alpha-\beta}[C]=$ $\mu^{\beta-\alpha} \cdot C$, substituting the function $v(x)=C$ into the boundary condition (18), for $f(x)=0$ we have

$$
C-\mu^{\beta-\alpha} \cdot C \sum_{k=1}^{\infty} a_{k}(x)=0, \quad x \in \partial \Omega .
$$

The last equality is equivalent to the equality

$$
C \cdot\left[1-\mu^{\beta-\alpha} \sum_{k=1}^{\infty} a_{k}(x)\right]=0 \text {. }
$$

We obtain from this the result that either $C=0$ or $\sum_{k=1}^{\infty} a_{k}(x)=\mu^{\alpha-\beta}$.

Thus, if conditions (19) and (20) are fulfilled, we obtain $C=0$, i.e. $v(x) \equiv 0$.

If the conditions (21) are fulfilled, then any constant is a solution of the homogeneous problem (17)-(18). In fact, substituting $v(x) \equiv C$ into equation (18), we obtain

$$
C-\sum_{k=1}^{\infty} a_{k}(x) \mu^{\beta-\alpha} C=C\left[1-\mu^{\beta-\alpha} \sum_{k=1}^{\infty} a_{k}(x)\right]=C\left[1-\mu^{\beta-\alpha} \mu^{\alpha-\beta}\right]=0 .
$$

The lemma is proved.

Now investigate existence of a solution of problem (17)-(18). Let $0 \leq \beta \leq \alpha$ and let $P(x, y)=\frac{1}{\omega_{n}} \frac{1-|x|^{2}}{|x-y|^{n}}$ be the Poisson kernel of the Dirichlet problem, and $\omega_{n}$ the area of the unit sphere.

Introduce the function

$$
P_{\alpha-\beta, \mu}(x, y)= \begin{cases}P(x, y), & \text { if } \alpha=\beta, \\ \frac{1}{\Gamma(\alpha-\beta)} \int_{0}^{1} s^{\mu-1}|\ln s|^{\alpha-\beta-1} P(s x, y) d s, & \text { if } \alpha>\beta,\end{cases}
$$

and consider the equation

$$
\psi(x)-\int_{\partial \Omega}\left[\sum_{k=1}^{\infty} a_{k}(y) P_{\alpha-\beta, \mu}\left(t_{k}(y), x\right)\right] \psi(y) d S_{y}=0 .
$$

The following statement holds.

Lemma 7 Let $\Gamma_{k} \subset \bar{\Omega}_{1} \subset \Omega, a_{k}(x), k=1,2, \ldots$, be continuous functions satisfying the condition (19). Then:

(1) If the condition (20) is realized, then problem (17)-(18) is uniquely solvable at any $f(x) \in C(\partial \Omega)$.

(2) If the condition (21) is realized, then problem (17)-(18) is solvable if the following condition is realized:

$$
\int_{\partial \Omega} f(x) \psi_{0}(x) d s_{x}=0
$$

where the function $\psi_{0}(x)$ is a solution of equation (23), moreover the number of independent solutions of this equation under these conditions is equal to 1. 
Proof Since $v(x)$ is a harmonic function, a solution of problem (17)-(18) can be found in the form of the Poisson integral $v(x)=\int_{\partial \Omega} P(x, y) v(x) d s_{x}$ where $v(x)$ is an unknown function. Substituting this function into the boundary condition (18), we obtain the integral equation with respect to the unknown function $v(x)$,

$$
v(x)-\int_{\partial \Omega}\left[\sum_{k=1}^{\infty} a_{k}(x) P_{\alpha-\beta, \mu}\left(t_{k}(x), y\right)\right] v(y) d S_{y}=f(x), \quad x \in \partial \Omega .
$$

Designate

$$
K(x, y)=-\sum_{k=1}^{\infty} a_{k}(x) P_{\alpha-\beta, \mu}\left(t_{k}(x), y\right) .
$$

Then equation (25) can be rewritten in the form of

$$
v(x)+\int_{\partial \Omega} K(x, y) v(y) d S_{y}=f(x), \quad x \in \partial \Omega .
$$

To investigate the solvability of the integral equation (26), we study the properties of the kernel $K(x, y)$. We show that $K(x, y)$ is a continuous function on $\partial \Omega \times \partial \Omega$.

In fact, since $t_{k}(x) \in \bar{\Omega}_{1} \subset \Omega$, we obtain $\left|s t_{k}(x)-y\right|>0$ for all $x, y \in \partial \Omega$, and therefore the function $P\left(s t_{k}(x), y\right)$ is continuous on $\partial \Omega \times \partial \Omega$. Further, the function $s^{\mu-1}|\ln s|^{\alpha-\beta-1}$ has an integrable singularity, and that is why the function $P_{\alpha-\beta, \mu}\left(t_{k}(x), y\right)$ is continuous on $\partial \Omega \times \partial \Omega$. Then by virtue of the uniform convergence of the series $\sum_{k=1}^{\infty} a_{k}(x)$, the kernel $K(x, y)$ is also a continuous function on $\partial \Omega \times \partial \Omega$.

Hence, one can apply Fredholm theory to equation (26). Since in the case of $f(x)=0$ and fulfillment of the condition (20), the solution of problem (17)-(18) can only be $v(x) \equiv 0$, for $f(x)=0$ the integral equation (26) has only a trivial solution.

Hence, for any $f(x) \in C(\partial \Omega)$ the solution of equation (26) exists, is unique, and belongs to the class $C(\partial \Omega)$. Using this solution, we construct the function $v(x)$ which will satisfy all the conditions of problem (17)-(18).

If the condition (21) is valid, then $v(x)=C$ satisfies the condition (18) at $f(x) \equiv 0$, i.e. the corresponding homogeneous equation (26) has the nonzero solution $v(x) \equiv C$. Then the adjoint homogeneous equation has also a nonzero solution, and that is why in this case fulfillment of the condition (24) is necessary and sufficient for solvability of problem (17)-(18). The lemma is proved.

\section{Study of the basic problem}

We now formulate the basic statement.

Theorem 1 Let $\mu>0,0 \leq \beta \leq \alpha, \alpha+\beta \neq 0, \Gamma_{k} \subset \bar{\Omega}_{1} \subset \Omega, a_{k}(x), k=1,2, \ldots$, be continuous functions satisfying the condition (19). Then:

(1) If the condition (20) is fulfilled, then problem (8)-(9) is uniquely solvable at any $f(x) \in C(\partial \Omega)$.

(2) If the condition (21) is fulfilled, then the condition (24) is necessary and sufficient for solvability of problem (8)-(9) where the function $\psi_{0}(x)$ is a solution of equation (23). If a solution of the problem exists, then it is unique up to the constant summand. 
(3) If a solution of problem (8)-(9) exists, then it is represented in the form of $u(x)=J_{\mu}^{\alpha}[v](x)$, where $v(x)$ is a solution of problem (17)-(18).

Proof (1) Let a solution $u(x)$ of problem (8)-(9) exist. Apply to this function the operator $D_{\mu}^{\alpha}$ and denote $v(x)=D_{\mu}^{\alpha}[u](x)$. Take the problem which the function $v(x)$ satisfies. Since by Lemma 2, in the case of harmonicity of the function $u(x)$, the function $D_{\mu}^{\alpha}[u](x)$ is also harmonic in $\Omega$, and the function $v(x)$ is harmonic.

Further, since according to Lemma 5 the equality $D_{\mu}^{\beta}[u](x)=J_{\mu}^{\alpha-\beta}\left[D_{\mu}^{\alpha}[u]\right](x)$ holds, the boundary condition of problem (8)-(9),

$$
D_{\mu}^{\alpha}[u](x)-\sum_{k=1}^{\infty} a_{k}(x) D_{\mu}^{\beta}[u]\left(t_{k}(x)\right)=f(x)
$$

with respect to the function $v(x)$ will be rewritten in the form of

$$
v(x)-\sum_{k=1}^{\infty} a_{k}(x) J_{\mu}^{\alpha-\beta}[v]\left(t_{k}(x)\right)=f(x), \quad x \in \partial \Omega
$$

In addition, since $D_{\mu}^{\alpha}[u](x) \in C(\bar{\Omega})$, we have $v(x) \in C(\partial \bar{\Omega})$. Thus, if $u(x)$ is a solution of problem (8)-(9), then the function $v(x)=D_{\mu}^{\alpha}[u](x)$ will be a solution of problem (17)-(18).

Now, let the conditions (19) and (20) be realized. Then by Lemmas 6 and 7, for any $f(x) \in$ $C(\partial \Omega)$ the solution of problem (17)-(18) exists, is unique, and designate $u(x)=J_{\mu}^{\alpha}[v](x)$, $x \in \bar{\Omega}$. Then we have by Lemma $\left.5 D_{\mu}^{\alpha}[u](x)=D_{\mu}^{\alpha} J_{\mu}^{\alpha}[v]\right](x)=v(x)$ in $\Omega$, and therefore we get $D_{\mu}^{\alpha}[u](x) \in C(\bar{\Omega})$. Harmonicity of the function $u(x)$ follows from Lemma 2 , and fulfillment of the conditions (9) can be checked immediately:

$$
\begin{aligned}
D_{\mu}^{\alpha}[u](x)-\sum_{k=1}^{\infty} a_{k}(x) D_{\mu}^{\beta}[u]\left(t_{k}(x)\right) \\
\quad=v(x)-\sum_{k=1}^{\infty} a_{k}(x) J_{\mu}^{\alpha-\beta}\left[D_{\mu}^{\alpha}[u]\right]\left(t_{k}(x)\right)=f(x), \quad x \in \partial \Omega .
\end{aligned}
$$

The first statement of the theorem is proved.

(2) Let now the condition (21) be fulfilled, and let the solution $u(x)$ of problem (8)-(9) exist. Consider the function $v(x)=D_{\mu}^{\alpha}[u](x)$. As in the first case, we show that the function $v(x)$ satisfies the conditions of problem (17)-(18). Then according to Lemma 7, fulfillment of the condition (24) is necessary. Thus, we prove the necessity of the condition (24) at fulfillment of the equality (21).

We show that if the equality (21) is fulfilled, then the condition (24) is also sufficient for the existence of the solution of problem (8)-(9).

In fact, if the conditions (21) and (24) are realized, a solution of problem (17)-(18) exists, is unique up to constant summand, and $v(x) \in C(\bar{\Omega})$. Then, similarly to the proof of the first statement of the theorem, the function $u(x)=J_{\mu}^{\alpha}[v](x)$ satisfies all the conditions of problem (8)-(9). The theorem is proved.

Remark 1 One can show that in the case of $\sum_{k=1}^{\infty}\left|a_{k}(x)\right|>\mu^{\alpha-\beta}$, the corresponding homogeneous problem (8)-(9) has nontrivial solutions. 
Example 1 Let $a_{1} \neq 0, a_{k}=0, k=2,3, \ldots$, and $t_{1}(x)=\delta x, 0<\delta<1$. Further, let $H_{k}(x)$ be a homogeneous harmonic polynomial of the power $k$. By virtue of the equality (11), we have $D_{\mu}^{\alpha}\left[H_{k}\right](x)=(k+\mu)^{\alpha} H_{k}(x)$.

Then

$$
\begin{aligned}
& H_{k}\left(t_{1}(x)\right)=H_{k}(\delta x)=\delta^{k} H_{k}(x), \\
& D_{\mu}^{\beta} H_{k}(\delta x)=\delta^{k}(k+\mu)^{\beta} H_{k}(x)
\end{aligned}
$$

and

$$
\begin{aligned}
D_{\mu}^{\alpha}\left[H_{k}\right](x)-a_{1} D_{\mu}^{\beta} H_{k}\left(t_{1}(x)\right) & =(k+\mu)^{\alpha} H_{k}(x)-a_{1} \delta^{k}(k+\mu)^{\beta} H_{k}(x) \\
& =\left[(k+\mu)^{\alpha}-a_{1} \delta^{k}(k+\mu)^{\beta}\right] H_{k}(x) .
\end{aligned}
$$

Hence, for $a_{1}=\delta^{-k}(k+\mu)^{\alpha-\beta}$ the harmonic polynomial $H_{k}(x)$ will be the solution of the homogeneous problem (8)-(9). If $\delta$ is a number close to zero, then we have $a_{1}>1$.

If the dimension of the space $n=3$, then the number of these polynomials is equal to $2 k+1[47]$.

\section{Competing interests}

The authors declare that they have no competing interests.

\section{Authors' contributions}

All authors completed the paper together. All authors read and approved the final manuscript.

\section{Acknowledgements}

This work has been supported by the MON Republic of Kazakhstan under Research Grant No. 0713/GF.

Received: 15 August 2013 Accepted: 9 January 2014 Published: 30 Jan 2014

\section{References}

1. Serbina, LI: A model of substance transfer in fractal media. Math. Model. 15, 17-28 (2003)

2. Koeller, RC: Application of fractional calculus to the theory of viscoelasticity. J. Appl. Mech. 51, 299-307 (1984)

3. Heymans, N, Podlubny, I: Physical interpretation of initial condition for fractional differential equations with Riemann-Liouville fractional derivatives. Rheol. Acta 45, 765-771 (2006)

4. Arena, P, Caponetto, R, Fortuna, L, Porto, D: Nonlinear Noninteger Order Circuits and Systems: an Introduction. World Scientific, River Edge (2000)

5. Bonilla, B, Rivero, M, Rodrigues-Germa, L, Trujillo, JJ: Fractional differential equations as alternative models to nonlinear differential equations. Appl. Math. Comput. 187, 79-88 (2007)

6. Amanov, D, Ashyralyev, A: Initial-boundary value problem for fractional partial differential equations of higher order. Abstr. Appl. Anal. (2012). doi:10.1155/2012/973102

7. Ashyralyev, A: A note on fractional derivatives and fractional powers of operators. J. Math. Anal. Appl. 357(1), 232-236 (2009)

8. Ashyralyev, A: Well-posedness of fractional parabolic equations. Bound. Value Probl. (2013). doi:10.1186/1687-2770-2013-31

9. Ashyralyev, A, Dal, F: Finite difference and iteration methods for fractional hyperbolic partial differential equations with the Neumann condition. Discrete Dyn. Nat. Soc. (2012). doi:10.1155/2012/434976

10. Ashyralyev, A, Dal, F, Pinar, Z: A note on the fractional hyperbolic differential and difference equations. Appl. Math. Comput. 217(9), 4654-4664 (2011)

11. Ashyralyev, A, Hicdurmaz, B: A note on the fractional Schrodinger differential equations. Kybernetes 40(5-6), 736-750 (2011)

12. Ashyralyev, A, Cakir, Z: FDM for fractional parabolic equations with the Neumann condition. Adv. Differ. Equ. (2013). doi:10.1186/1687-1847-2013-120

13. Yakar, A, Koksal, ME: Existence results for solutions of nonlinear fractional differential equations source. Abstr. Appl. Anal. (2012). doi:10.1155/2012/267108

14. Tikhonov, AN: On boundary conditions containing derivatives of order higher than the order of the equation. Mat. Sb. 26(1), 35-56 (1950) (Russian)

15. Amanov, D, Yuldasheva, AV: Solvability and spectral properties of boundary value problems for equations of even order. Malays. J. Math. Sci. 3(2), 227-248 (2009) 
16. Amanov, D, Murzambetova, MB: A boundary value problem for a fourth order partial differential equation with the lowest term. Vestn. Udmurtsk. Univ. Mat. Mekh. Komp. Nauki 2013(1), 3-10 (2013) (Russian)

17. Amanov, D: Boundary value problem for the higher order equation with fractional derivative. Malays. J. Math. Sci. 7(2), 203-217 (2013)

18. Bavrin, II: Operators for harmonic functions and their applications. Differ. Uravn. 21, 9-15 (1985) (Russian)

19. Sokolovskii, VB: On a generalization of the Neumann problem. Differ. Uravn. 24, 714-716 (1988) (Russian)

20. Bitsadze, AV: On the Neumann problem for harmonic functions. Dokl. Akad. Nauk SSSR 311, 11-13 (1990) (Russian)

21. Berdyshev, AS, Turmetov, BK, Kadirkulov, BJ: Some properties and applications of the integrodifferential operators of Hadamard-Marchaud type in the class of harmonic functions. Sib. Math. J. 53(4), 600-610 (2012) (Russian)

22. Kirane, M, Tatar, NE: Nonexistence for the Laplace equation with a dynamical boundary condition of fractional type. Sib. Math. J. 48(5), 1056-1064 (2007) (Russian)

23. Torebek, BT, Turmetov, BK: On solvability of a boundary value problem for the Poisson equation with the boundary operator of a fractional order. Bound. Value Probl. 2013, 93 (2013). doi:10.1186/1687-2770-2013-93

24. Turmetov, BK: On a boundary value problem for a harmonic equation. Difer. Uravn. 32(8), 1089-1092 (1996) (Russian)

25. Turmetov, BK: On smoothness of a solution to a boundary value problem with fractional order boundary operator. Mat. Tr. 7(1), 189-199 (2004) (Russian)

26. Umarov, SR, Luchko, YF, Gorenflo, R: On boundary value problems for elliptic equations with boundary operators of fractional order. Fract. Calc. Appl. Anal. 3(4), 454-468 (2000)

27. Samko, SG, Kilbas, AA, Marichev, Ol: Fractional Integrals and Derivatives, Theory and Applications. Gordon \& Breach, Amsterdam (1993)

28. Kilbas, AA, Tityura, AA: Fractional derivative of the Marsho-Hadamard type and inversion of fractional integrals. Dokl. Nat. Akad. Nauk Belarusi 50(4), 5-10 (2006) (Russian)

29. Bitsadze, AV, Samarskii, AA: Some elementary generalizations of linear elliptic boundary value problems. Dokl. Akad. Nauk SSSR 185, 739-740 (1969) (Russian)

30. Pulatov, AK: A Bitsadze-Samarskii problem. Differ. Uravn. 25, 537-540 (1989) (Russian)

31. Skubachevskii, AL: Nonclassical boundary value problems. I. Sovrem. Mat. Fundam. Napravl. 26, 3-132 (2007) (Russian)

32. Skubachevskii, AL: Nonclassical boundary value problems. II. Sovrem. Mat. Fundam. Napravl. 33, 3-179 (2009) (Russian)

33. Ashyralyev, A: On well-posedness of the nonlocal boundary value problem for elliptic equations. Numer. Funct. Anal. Optim. 24(1-2), 1-15 (2003). doi:10.1081/NFA-120020240

34. Ashyralyev, A: A note on the Bitsadze-Samarskii type nonlocal boundary value problem in a Banach space. J. Math. Anal. Appl. 344(1), 557-573 (2008)

35. Ashyralyev, A, Sharifov, YA: Existence and uniqueness of solutions of the system of nonlinear fractional differential equations with nonlocal and integral boundary conditions. Abstr. Appl. Anal. (2012). doi:10.1155/2012/594802

36. Ashyralyev, A, Ozger, F: The hyperbolic-elliptic equation with the nonlocal condition. Math. Methods Appl. Sci. (2013). doi:10.1002/mma.2811

37. Ashyralyev, A, Ozturk, E: The numerical solution of the Bitsadze-Samarskii nonlocal boundary value problems with the Dirichlet-Neumann condition. Abstr. Appl. Anal. (2012). doi:10.1155/2012/730804

38. Ashyralyev, A, Ozturk, E: On Bitsadze-Samarskii type nonlocal boundary value problems for elliptic differential and difference equations: well-posedness. Appl. Math. Comput. 219(3), 1093-1107 (2012)

39. Ashyralyev, A, Tetikoglu, FS: A note on Bitsadze-Samarskii type nonlocal boundary value problems: well-posedness. Numer. Funct. Anal. Optim. 34(9), 939-975 (2013)

40. Berdyshev, AS, Kadirkulov, BJ, Nieto, JJ: Solvability of an elliptic partial differential equation with boundary condition involving fractional derivatives. Complex Var. Elliptic Equ. (2013). doi:10.1080/17476933.2013.777711

41. Karachik, W, Turmetov, BK, Torebek, BT: On some integro-differential operators in the class of harmonic functions and their applications. Sib. Adv. Math. 22(2), 115-134 (2012)

42. Mahmudov, NI, Zorlu, S: Approximate controllability of fractional integro-differential equations involving nonlocal initial conditions. Bound. Value Probl. (2013). doi:10.1186/1687-2770-2013-118

43. Qu, H, Liu, X: Existence of nonnegative solutions for a fractional $m$-point boundary value problem at resonance. Bound. Value Probl. 2013, 127 (2013). doi:10.1186/1687-2770-2013-127

44. Hilmi, E, Kilicman, A: Nonlocal boundary value problems for impulsive fractional integro-differential equations in Banach spaces. Bound. Value Probl. 2012, 145 (2012). doi:10.1186/1687-2770-2012-145

45. Stein, EM, Weiss, G: Introduction to Fourier Analysis on Euclidean Spaces. Princeton University Press, Princeton (1971)

46. Vladimirov, VS: Uravneniya mathematicheskoi fiziki. Nauka, Moscow (1981) (Russian)

47. Bitsadze, AV: Uravneniya mathematicheskoi fiziki. Nauka, Moscow (1982) (Russian)

10.1186/1687-2770-2014-29

Cite this article as: Muratbekova et al.: On solvability of a nonlocal problem for the Laplace equation with the fractional-order boundary operator. Boundary Value Problems 2014, 2014:29 